\title{
Local phase control for a planar array of fiber laser amplifiers
}

Patrick Steffanic $^{\mathrm{a}}$, Benjamin T. Johannes ${ }^{\mathrm{a}}$, Claudia A. Sison ${ }^{\mathrm{b}}$, Gary B. Hughes ${ }^{\mathrm{b}}$, Philip Lubin $^{c}$, Peter Meinhold ${ }^{c}$, Jonathan Suen ${ }^{c}$, Hugh O’Neill ${ }^{d}$, Miikka Kangas ${ }^{\mathrm{c}}$, Travis Brashears $^{\mathrm{c}}$, Qicheng Zhang ${ }^{\mathrm{c}}$, Janelle Griswold ${ }^{\mathrm{c}}$, Jordan Riley ${ }^{\mathrm{c}}$ and Caio Motta ${ }^{\mathrm{c}}$ psteffan@calpoly.edu gbhughes@calpoly.edu

aPhysics Department, California Polytechnic State University, San Luis Obispo, CA 93407-0405

bStatistics Department, California Polytechnic State University, San Luis Obispo, CA 93407-0405

${ }^{\mathrm{c}}$ Physics Department, University of California, Santa Barbara, CA 93106-9530

${ }^{\mathrm{d}}$ Physics Department, Ventura College, Ventura, CA 93003

\begin{abstract}
Arrays of phase-locked lasers have been developed for numerous directed-energy applications. Phased-array designs are capable of producing higher beam intensity than similar sized multi-beam emitters, and also allow beam steering and beam profile manipulation. In phased-array designs, individual emitter phases must be controllable, based on suitable feedback. Most current control schemes sample individual emitter phases, such as with an array-wide beam splitter, and compare to a master phase reference. Reliance on a global beam splitter limits scalability to larger array sizes due to lack of design modularity. This paper describes a conceptual design and control scheme that relies only on feedback from the array structure itself. A modular and scalable geometry is based on individual hexagonal frames for each emitter; each frame cell consists of a conventional lens mounted in front of the fiber tip. A rigid phase tap structure physically connects two adjacent emitter frame cells. A target sensor is mounted on top of the phase tap, representing the local alignment datum. Optical sensors measure the relative position of the phase tap and target sensor. The tap senses the exit phase of both emitters relative to the target normal plane, providing information to the phase controller for each emitter. As elements are added to the array, relative local position data between adjacent phase taps allows accurate prediction of the relative global position of emitters across the array, providing additional constraints to the phase controllers. The approach is scalable for target distance and number of emitters without loss of control.
\end{abstract}

Keywords: DE-STAR, Directed Energy, Laser Phased Array, Planetary Defense

\section{INTRODUCTION}

\subsection{DE-STAR System Concept}

Recent studies have considered the use of directed energy for planetary defense against asteroid impact. High-intensity illumination is directed at a threatening asteroid, evaporating surface material to create a reactionary force that alters the asteroid's orbit. Directed Energy System for Targeting of Asteroids and exploRation (DE-STAR) is a conceptual orbiting platform consisting of a planar array of laser fiber amplifiers and associated optical elements, and powered by photovoltaics. ${ }^{1,2}$ The main objective of DE-STAR is to use directed energy to deliver sufficient photon flux to vaporize a spot on the surface of an asteroid. A sufficiently large DE-STAR array could target near-Earth asteroids (NEAs) from low-Earth orbit. Smaller arrays would be useful for missions that deploy to an area near the asteroid, deemed DE-STARLITE. ${ }^{3,4}$ An operational DE-STAR or DE-STARLITE would be capable of serving diverse scientific objectives, including spacecraft propulsion ${ }^{5}$, active illumination for asteroid search and orbit refinement ${ }^{6}$, stand-off composition analysis ${ }^{7}$, orbital debris removal and more; multiple uses of the DE-STAR system are depicted in Fig. 1.

The design concept of DE-STAR is an array of phase-locked laser fiber amplifiers. A concentrated directed energy beam is formed through

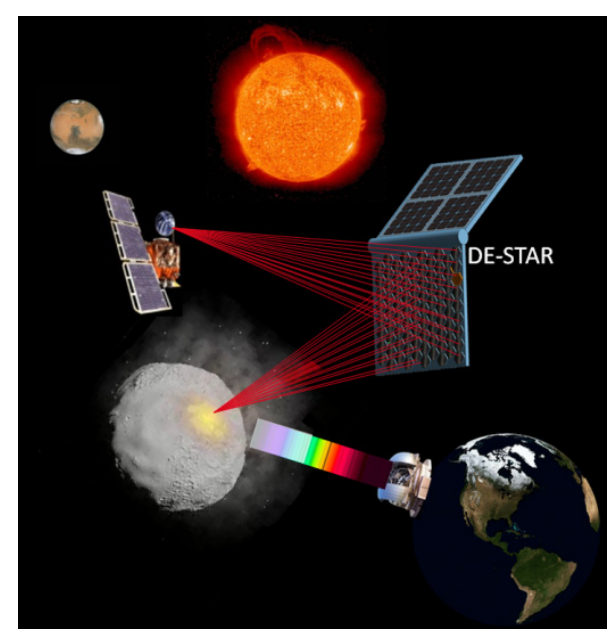

Figure 1. DE-STAR, a proposed laser phased array system for planetary defense and other scientific purposes. 
precise phase control of individual laser emitters, with phase-locking of among all emitters across the entire array. Beams from individual emitters need to coalesce at the target, and the phases of each individual beam need to align so that constructive interference occurs, i.e., the combination of individual beams produces an amplified signal at the target.

\subsection{Laser Phased Array Concept}

The performance of Ytterbium-doped fiber laser amplifiers (YDFA) has improved markedly in recent years. Continuous-wave, multi-kW-class devices are now routine and affordable, germinating many novel applications. ${ }^{8}$ Phased array configurations of laser fiber amplifiers have been demonstrated in the laboratory. ${ }^{9,10,11,12,13}$ A phased array design requires a control scheme to ensure that individual beams are in phase in the far-field. In a standard approach, heterodyning systems employ phase feedback based on sampling and comparing the output phase of each emitter to a global reference phase, such as produced by a master oscillator. ${ }^{14,15,16}$ Innovative control schemes such as Stochastic Parallel Gradient Decent (SPGD) method combine multiple feedback beams at a single photodetector. ${ }^{17,18,19,20}$ A conceptual phased array design with SPGD control is depicted in Fig. 2. In a SPGD control scheme, phases of individual emitters are adjusted iteratively until the combined signal at the photodetector is maximized. Fielddeployable systems have also been developed that include object-in-the-loop control to compensate for atmospheric turbulence. ${ }^{21,22}$

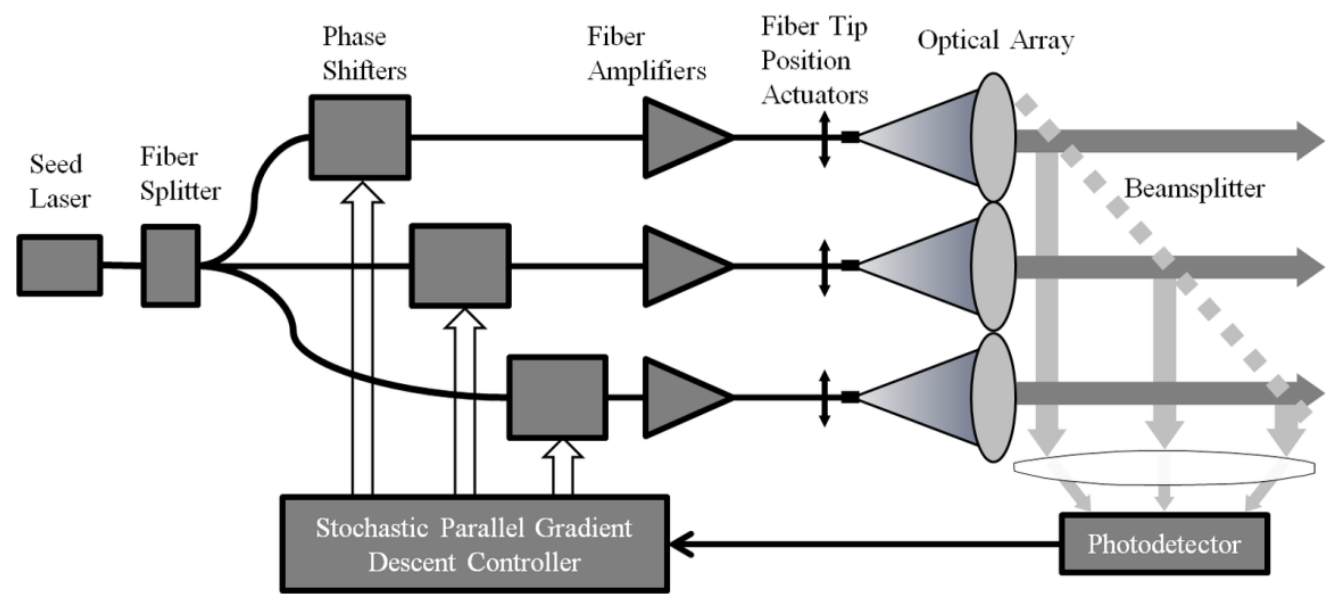

Figure 2. Stochastic Parallel Gradient Descent (SPGD) control scheme used in existing laser phased array laser systems, based on high-power laser fiber amplifiers.

\subsection{Motivation for Local Control Scheme}

As depicted in Fig. 2, the SPGD control scheme employs a beamsplitter to sample the outgoing phase of each individual emitter. The design requires the beamsplitter to be optically flat over the combined aperture of the overall array. Alternatively, structural aberrations in the beamsplitter could be characterized during a calibration procedure, and compensations could be implemented in the controller. Such a calibration procedure is capable of compensating for static anomalies in the beamsplitter. It may also be possible for the controller to compensate for some transient aberrations that mutate more slowly than the minimum control response time. These disadvantages are tenable when the objective is to build an array with a fixed number of emitters. That is, careful construction of the array and programming of the controller can readily compensate for optical imperfections in system components. A DE-STARLITE mission could be designed around a fixed-sized array, using current phased array designs.

The DE-STAR concept envisions a more modular approach, whereby the emitter array can be constructed in phases by adding individual emitters around the periphery of a growing array. Based on current designs, if an emitter were added to the array, the beamsplitter would need to be expanded to cover the new array aperture. Replacing an existing beamsplitter with a larger one, or adding a segment to an existing beamsplitter, to cover the additional emitter would require a re-calibration of the added emitter to the rest of the array. In the context of an orbiting platform such as DE-STAR, re-calibration of the control components is impractical.

A novel concept for a phased array design and control scheme employs hexagonal emitter cell units that include a phase tap structure. Unit cells are built and calibrated in the factory, and the modular design supports mechanical attachment of additional emitter cells to an existing array, without the need to modify or re-calibrate any aspect of existing emitter components. As an emitter is added to an existing array, the control module $\mathrm{I} / \mathrm{O}$ is updated to 
accommodate the additional sensors and controls. The phase perturbations in individual beams that must be counteracted, namely fast structural vibration and slow thermal drift, can be measured and corrected for with electronic phase control in the fiber amplifier chain. A local control scheme is independent of target and range and is more easily scalable in both power and array size. Structural vibrations in the first, second, and third modes may have large enough amplitudes to deflect the beam away from the target. To counteract these vibrations a micropositioner will be employed to slightly bend the fiber tip so as to keep it locked on target. In addition, the structure of each beam's cell has been optimized to reduce first, second, and third mode amplitudes as much as possible.

The core idea of the new design is that the relative phases of adjacent beams are determined with respect to a target plane. The relative position between adjacent phase sensors is determined with respect to a mechanical datum that is located at the center of a target sensor. Calibration of individual emitters is accomplished during module assembly, and calibration data is added to the array controller. This paper describes a conceptual mechanical design and phase control scheme for beam formation and steering in the context of a directed-energy system consisting of a planar array of phase-locked lasers. The mechanical design and phase control concept are presented in the following sections.

\section{MECHANICAL DESIGN AND LOCAL PHASE CONTROL CONCEPT}

\subsection{Mechanical Components}

The overall array is built up from individual emitter cells. Each emitter cell consists of a hexagonal frame that supports a conventional optic (Fig. 3). Alternatively, a thin-film holographic lens with equivalent optical characteristics could be used. ${ }^{23}$ Optical component dimensions are driven by mission parameters. A hypothetical mission is envisioned where the array is deployed to a position near the target, such as DE-STARLITE. Hypothetical mission parameters include production of a beam from each emitter that is $1 \mathrm{~m}$ diameter on the surface of a target that is $10 \mathrm{~km}$ away $(0.1 \mathrm{~m}$ rad field of view). With adequate pointing and phase control, individual beams will combine within the $1 \mathrm{~m}$ spot to produce a $\sim 10 \mathrm{~cm}$ spot with highly amplified beam intensity.

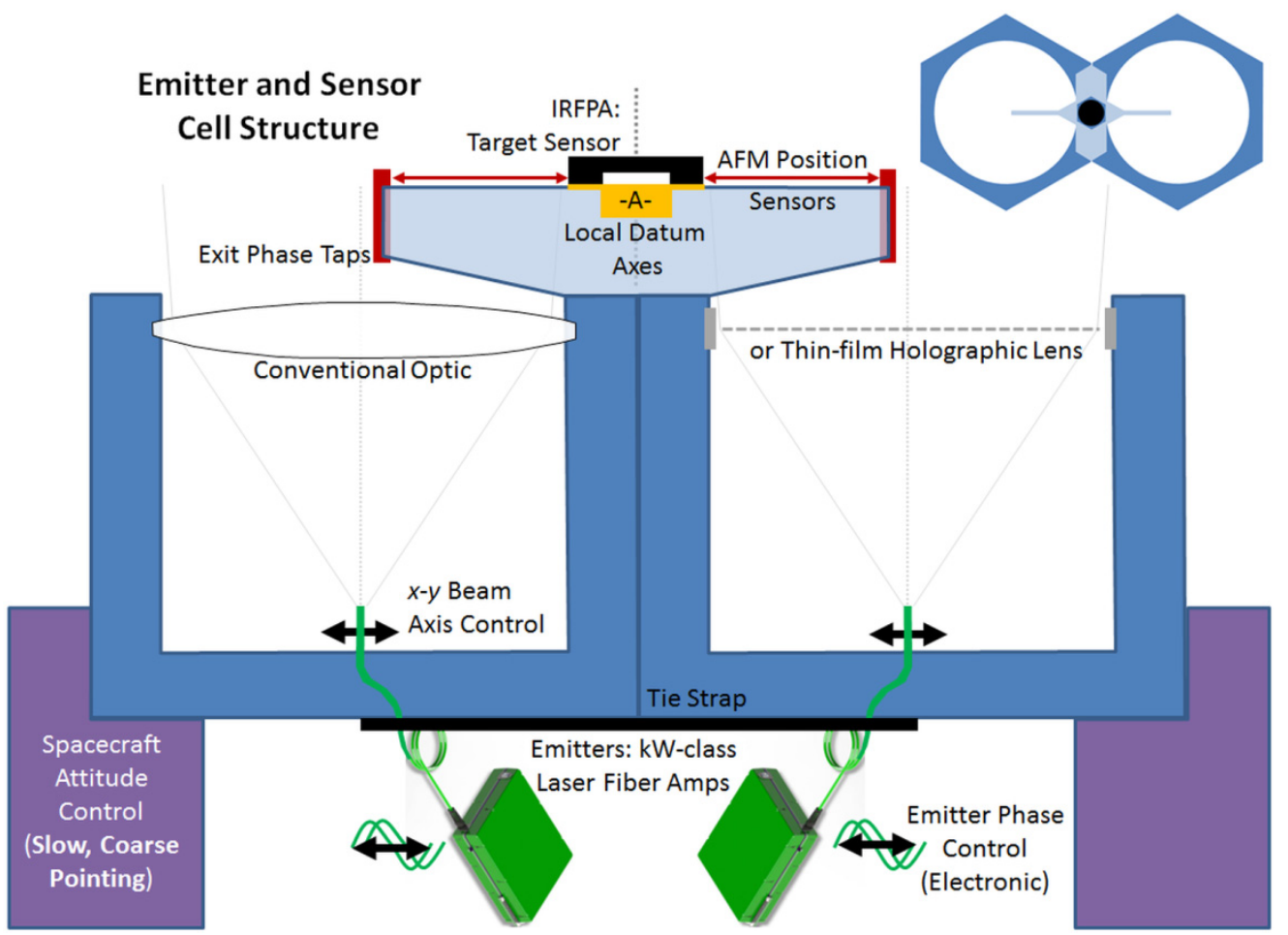

Figure 3. Conceptual diagram of a 2-element laser phased array architecture. A single phase tap structure connects the two emitter cells. Datum -A- is a mechanical reference location that provides a common coordinate system for the target vector and the phase control mechanism. Mission parameters drive the optical configuration. For a stand-on mission, such as DE-STARLITE, an example might be to seek a $1 \mathrm{~m}$ beam on a target that is $10 \mathrm{~km}$ distant, leading to individual emitter lenses that are $\sim 55 \mathrm{~mm}$ diameter. 
The requirement for $0.1 \mathrm{~m}$ rad field of view establishes the mechanical characteristics of the emitter array. The emitter diameter (fiber diameter) is $\approx 12.5 \mu \mathrm{m}$ with a numerical aperture of $\approx 0.22$. The focal length of the lens is:

$$
f=\frac{D_{\text {emitter }}}{2 \cdot \tan \left(\frac{1}{2} \cdot F O V_{\text {lens }}\right)} \approx 0.125 \mathrm{~mm}
$$

The $\mathrm{F} \#$ of the lens is determined by matching numerical aperture of the emitter in a medium with refractive index $n=1$ :

$$
F \#=\frac{1}{2 \cdot \operatorname{asin}\left(\frac{N A_{\text {emitter }}}{n}\right)} \approx 2.254
$$

The lens diameter for an array cell that produces a beam $1 \mathrm{~m}$ diameter on the surface of a target that is $10 \mathrm{~km}$ away $(0.1 \mathrm{~m}$ rad field of view) in a medium with refractive index $n=1$ is:

$$
D_{\text {lens }}=\frac{f}{F \#} \approx 55.45 \mathrm{~mm}
$$

The entire emitter array structure is supported by a connecting frame, which includes components that provide navigation, guidance and control for the overall array. Spacecraft attitude sensing and control is based on conventional celestial guidance principles, and provides coarse pointing that can be much slower than the beam axis and phase control feedback rates. In the example presented here $(0.1 \mathrm{~m}$ rad field of view), the rough pointing requirement provided by spacecraft attitude control is estimated to be on the order of $\sim 10 \mathrm{~m} \mathrm{rad}$. The requirement is driven by the range of motion of $x-y$ emitter positioner that directs the beam axis of each emitter.

Between adjacent emitter cells is a rigid phase tap structure that measures the output phase of each adjacent beam, as shown in Fig. 3. An infrared target sensor is mounted on top of the phase tap structure, and provides a datum to link mechanical features in the emitter cell to the target vector. The emitter fiber is accommodated through the base of the hexagonal frame. The emitter fiber is secured within the frame by a 2-axis $(x-y)$ micro-positioner. Moving the emitter tip laterally behind the optic alters the beam axis. Emitter tip adjustments are used to keep the individual beam axes directed toward the same spot on the target, ensuring that beams coalesce in the far field. Beam axis adjustments are characterized as intermediate pointing. The main requirement is to keep the beams from each emitter pointed to the same point on the target, so that the overlapping beams coalesce in the center, allowing constructive interference to create an amplified signal in the center of the beams. Using the rough requirement that beams should not drift more than one-tenth the beam diameter, the intermediate pointing requirement imposed on the fiber tip micropositioners is on the order of $\sim 10 \mu$ rad in the example presented here. Emitter tip adjustments are independent of the phase control feedback scheme, but must occur rapidly enough to counteract low-mode vibrations in the emitter array structure that cause the emitter beam axis to wander. $Z$-axis movements of the emitter tip are not required for phased-array operation, because individual beams do not need to be focused on the target in the conventional sense. Rather, when multiple beams coalesce at the target, i.e., the beam axes are all pointed at roughly the same spot on the target and the beams overlap, then flux delivered to the target is maximized whenever all the beam phases are aligned. So, the only requirements for phased-array operation are beam axis alignment and phase alignment. The emitter tip control scheme is significantly simplified without requirements for $z$-axis alignment. Individual emitters are $\mathrm{kW}$-class laser fiber amplifiers. A key feature of such amplifiers is that the output phase can be controlled electronically, eliminating the need to physically move the fiber tip in the $z$-axis direction. Alternatively, phase control could be accomplished by controlling each fiber tip in a hexapod with full six degree-of-freedom (DOF) positional movements available. Finer beam pointing and beam steering can be accomplished by phase steering, i.e., adjusting the relative phases of adjacent emitters to produce a peak combined amplitude at a specified point within the overall beam diameter.

\subsection{Phase Alignment Concept}

As with many optical systems, phase alignment for a laser array must be on the order of $\lambda / 10$ for quality beam formation. ${ }^{24}$ Achieving adequate phase control requires attention to several key aspects of system design that can affect the relative phases of every emitter. Accurate target acquisition and pointing with respect to a mechanical datum in the emitter array is required. The target vector establishes a phase reference plane, which is normal to the target vector, as depicted in Fig. 4. The output phase of every emitter must be controlled with respect to the phase reference plane. Mechanical calibration and relative alignment of optical and mechanical components is necessary. Due to structural vibration, the relative position of optical and mechanical components must be determined within the required control 
feedback time ('control-time') for closed-loop emitter phase control. YDFA drift, thermal changes in the fiber and other sources of phase perturbation are accounted for by sensing the relative phase beyond the exit aperture.

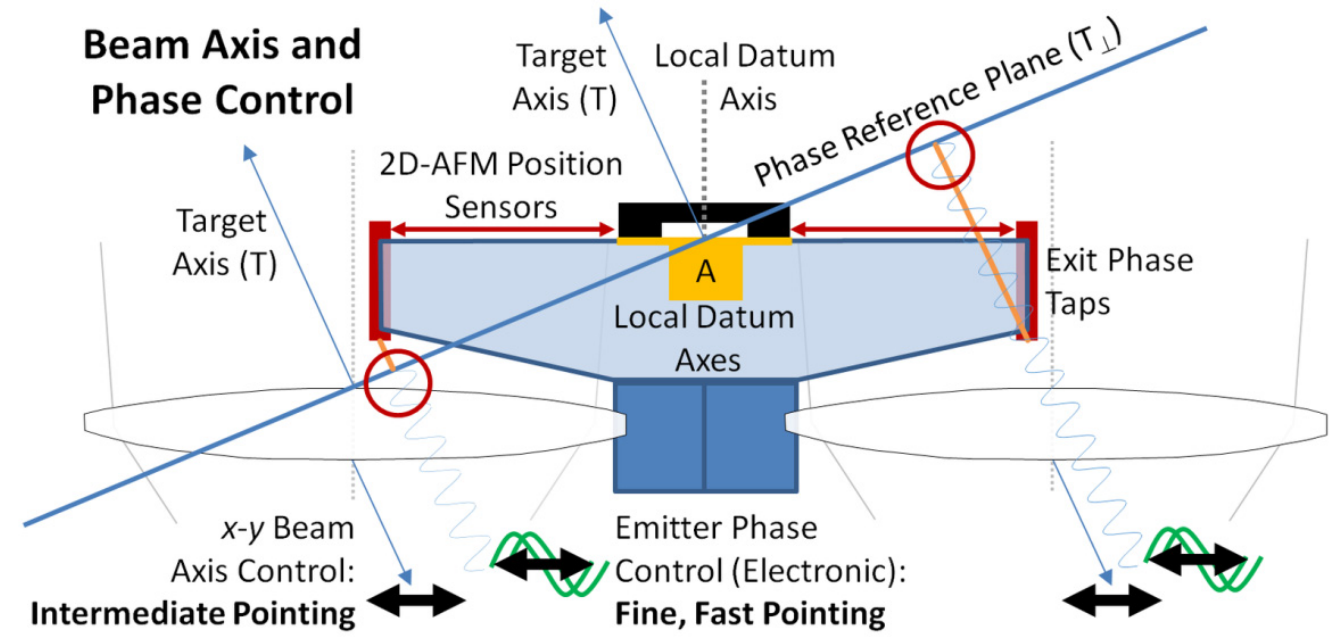

Figure 4. Conceptual diagram of a phase tap structure illustrating the mechanism for sensing the phase of individual elements in relation to the phase reference plane. The phase reference plane is derived from the target vector, using camera and sensor calibration information. The position of each exit phase tap in the mechanical datum coordinate system is measured with 2D-AFM position sensors (shown in Fig. 5). The calculated distance from the phase tap to the reference plane along the target vector provides the reference phase for aligning the two emitter phases.

\subsection{Camera Calibration and Target Axis in Datum Coordinate System}

The target is viewed using an infrared imaging system, consisting of an optic and an infrared focal plane array (IRFPA) that are mounted together in a mechanical housing. Target acquisition algorithms determine the target centroid $\left\langle x_{c}, y_{c}\right\rangle$ in the IRFPA pixel array coordinate system. ${ }^{25}$ Using camera calibration information, the target centroid $\left\langle x_{c}, y_{c}\right\rangle$ is then converted to a target axis $\left\langle\theta_{T}, \varphi_{T}\right\rangle$ as polar $(\theta)$ and azimuthal $(\varphi)$ directions in the infrared camera mount mechanical datum coordinate system, using transformations deduced from innovative calibration algorithms. ${ }^{26,27,28}$ Conversion from image coordinates to the target axis is accomplished using a composite inverse transformation: ${ }^{29}$

$$
\left[\begin{array}{l}
\theta_{T} \\
\varphi_{T}
\end{array}\right]=\left(A^{\circ} D^{\circ} F\right)^{-1}\left(\left[\begin{array}{l}
x_{c} \\
y_{c}
\end{array}\right]\right)
$$

where $F$ is the transformation from the target axis to ideal pixel coordinates, $D$ is a lens distortion mapping, and $A$ is the ideal affine transformation from scene coordinates to pixel coordinates, all determined during camera calibration.

The relationship between pixel coordinates and the mechanical mounting is also characterized during fabrication. Precision machining is not adequate for ultra-precision alignment, but statistical calibration/characterization techniques are available. ${ }^{30}$ The statistical approach reduces measurement error and supports ultra-precision alignment with 'irregular' machined datum surfaces.

\subsection{Phase Tap Position in Datum Coordinate System}

In order to control the phase of each emitter at the target plane, the distance from each phase tap to the target plane must be determined within the required control-time. The phase tap positions within the datum coordinate system are determined using technology developed for atomic force microscopes (AFMs). In an AFM, a molecular tip is mounted on the free end of a cantilever. As the tip is moved across the surface of a sample, molecular-scale movements of the cantilever are induced. A laser is reflected off the back side of the cantilever, and detected at a split photodiode. Movements of the cantilever are detected in the changing position of the laser spot on the photodiode. The AFM cantilever provides a $\sim$ three DOF measurement system (although typical AFM systems only sense one or perhaps 2 of the potential movements). The mechanism is extended to six-DOF measurement by using an array of lasers, and by including a curved reflective surface such as a hyperbolic paraboloid ${ }^{31}$ (Fig. 5). The reflected spots are sensed at 2dimensional photodiode arrays in the emitter plane. Changes in the relative position of the emitter plane and reflective surface will shift the location of the reflected spots within photodiode arrays. All six degrees of freedom produce 
independent shifts in the reflected spot locations, allowing full six-DOF relative position determination. The sensors are used to make control-time measurements of the position of the exit phase taps within the local mechanical datum coordinate system.

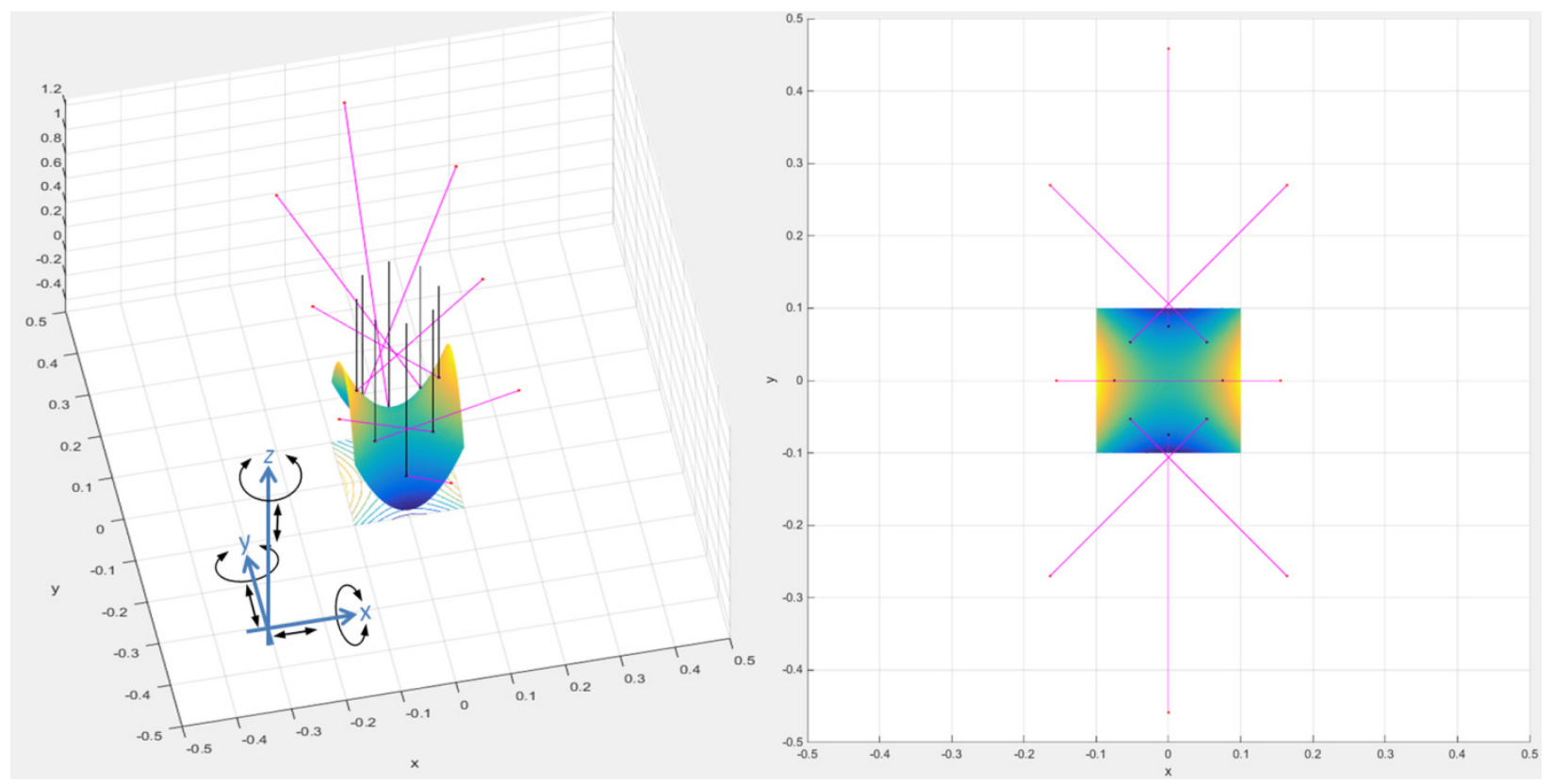

Figure 5. Conceptual diagram of a six-DOF relative position measurement scheme, which is a 2-dimensional extension of Atomic Force Microscope (AFM) technology. ${ }^{31}$ Laser emitters are directed at a curved reflective surface; reflected spots are sensed in 2-D photodiode arrays in the emitter plane. Changes in the relative position of the emitter plane and reflective surface will shift the location of reflected spots.

Kinematic motions and independent changes in the spot pattern ensure that position determination is invertible for simultaneous motions in all degrees of freedom, i.e., for some measured change in the spot location from the nominal $\left\langle\Delta x, \Delta y, \Delta z, \Delta \theta_{x}, \Delta \theta_{y}, \Delta \theta_{z}\right\rangle$, the relative position of the emitter plane and reflective plane can be determined. The emitter plane maintains a rigid spatial relationship within the mechanical datum coordinate system, and the phase tap maintains a rigid spatial relationship to the reflective plane. Using the relative position of the reflective plane, and the rigid relationships, the position of the phase tap within the mechanical datum coordinate system is determined.

The target axis $\left\langle\theta_{T}, \varphi_{T}\right\rangle$ in the mechanical datum coordinate system is used to establish the target plane, which (arbitrarily) passes through the datum origin. The plane becomes the phase reference plane, which is:

$$
N_{T} \cdot\langle x, y, z\rangle=0, \quad N_{T}=\frac{\left\langle\cos \left(\theta_{T}\right) \sin \left(\varphi_{T}\right), \sin \left(\theta_{T}\right) \sin \left(\varphi_{T}\right), 1\right\rangle}{\left\|\left\langle\cos \left(\theta_{T}\right) \sin \left(\varphi_{T}\right), \sin \left(\theta_{T}\right) \sin \left(\varphi_{T}\right), 1\right\rangle\right\|}
$$

A single phase tap structure connects two adjacent emitters. A nominal location of the phase taps within the mechanical datum coordinate system are determined during assembly calibration. The target plane is the phase reference plane: the control system must seek to align the phases of all emitters to the same value at the target plane. Deviations from the calibrated (mechanical datum) location of the phase taps are determined in control time with AFM relative position sensors. The distance of the phase taps from the phase reference plane can then be determined.

$$
D_{\text {ref }}=\frac{N_{T} \cdot\left\langle x_{\text {tap }}, y_{\text {tap }}, z_{\text {tap }}\right\rangle}{\left\|N_{T}\right\|}=N_{T} \cdot\left\langle x_{\text {tap }}, y_{\text {tap }}, z_{\text {tap }}\right\rangle
$$

Distance along the target vector from the phase tap provides the phase alignment target at the phase tap. Phase alignment is modulo one cycle, e.g., shift the measured phase $\phi_{\text {meas }}$ of an emitter to zero in the phase alignment plane:

$$
\Delta \phi=\phi_{\text {meas }}-\frac{\frac{D_{\text {ref }}}{\lambda}-\left\lfloor\frac{D_{\text {ref }}}{\lambda}\right\rfloor}{2 \pi}
$$




\subsection{Adding Emitters to the Array}

A single phase tap structure connects two adjacent emitters, and establishes a datum coordinate system for relative phase alignment of the two emitters in the target plane. When a third emitter is added to the array, two additional phase tap structures are also added, as depicted in Fig. 6.

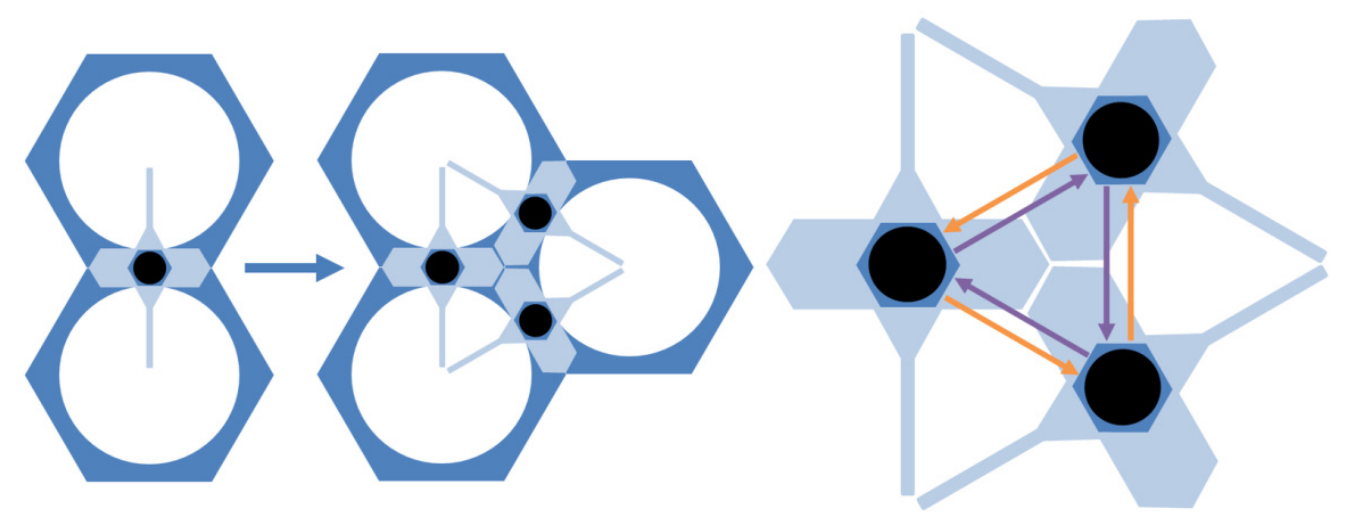

Figure 6. Conceptual diagram of three adjacent cells of a laser phased array. Three phase tap structures are used to connect the adjacent cells. The relative position between adjacent phase tap structures is determined with 2D-AFM position sensors (shown in Fig. 5).

Each additional emitter cell is installed with two pre-calibrated phase tap structures. The phase tap structures are calibrated in the factory so that the nominal positions of the phase taps are known within the mechanical datum coordinate system. Each phase tap structure will have an independent datum coordinate system, and the relationship between two adjacent phase taps must be determinable in control-time. The relative position between adjacent phase tap structures is determined with 2D-AFM position sensors (shown in Fig. 5). The nominal position of the existing tap with respect to the two added taps is determinable with angle-side-angle relationships. The array core is then built up by adding emitter cells to the periphery of the existing array, as shown in Fig. 7.

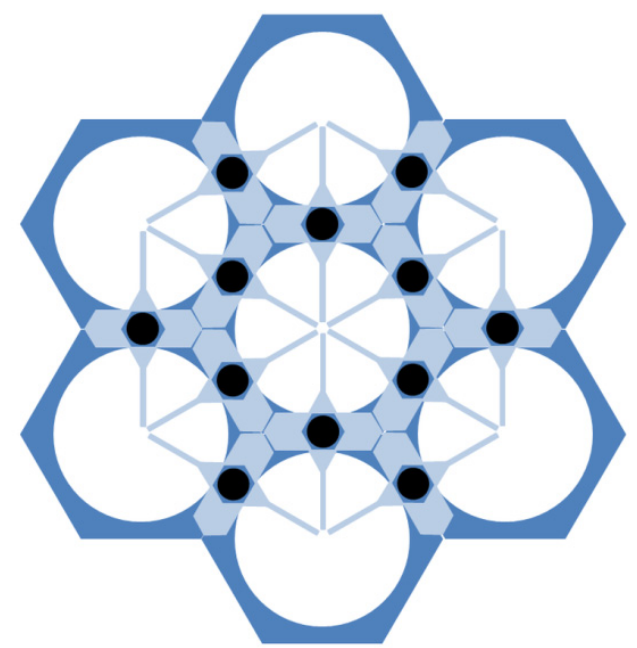

Figure 7. Conceptual diagram of adjacent cells of a laser phased array. The array core is built up by adding emitter cells to the periphery of the existing array.

The design is modular and extensible. A frame for an emitter cell that is to be added to the array is fabricated. Two hexagonal tie plates are installed on the bottom of an emitter frame. The fiber amplifier module is mounted on a tie plate, and the fiber is secured on the inside of the frame by installing the fiber tip positioners. The optic is then installed over the face of the frame. Two phase tap structures are installed on the top of the frame, and calibration procedures are performed at the factory, to characterize the nominal relationships between camera coordinates, IRFPA coordinates, the mechanical datum locations and the phase tap locations. Mechanical connection to the existing array is accomplished by 
bolting the free edge of the two phase tap structures to the mating emitter cells in the existing array, and by securing the two hexagonal tie straps on the base to the adjacent cell frames.

When an emitter cell is added to the array, two additional phase taps are also added (Fig. 6). The two additional taps also contain two new IRFPA target sensors. Each target sensor will generate a target vector in its own mechanical datum coordinate system. A resultant target vector must be determined from the combined information of all individual target sensors, and within a common coordinate system. The relationship between the mechanical datums of two adjacent target sensors is determined by 6-DOF position sensors. A virtual coordinate system is established with its origin in the center of the emitter array, and provides a global reference plane. The global coordinate system is established by combining the relative positions of all target sensors. The position of each phase tap sensor in the global coordinate system is determinable from the global position of the associated target sensor mount.

\section{CONTROL SCHEME AND BEAM SIMULATION}

\subsection{Coarse, Intermediate and Precision Pointing, and Beam Steering}

The preceding discussion of the emitter array architecture forms the basis for a phase feedback control scheme. In this paper, it is assumed that spacecraft attitude control ('rough pointing', $\sim 10 \mathrm{~m}$ rad in the example presented above) is adequate, and does not introduce significant pointing jitter into the target acquisition sensors. The 'rough pointing' control system operates independently from the 'intermediate pointing' (beam axis) and 'fine pointing' (beam steering) control systems. The intermediate and fine control systems are based on sensors consisting of phase taps, 6-DOF position sensors and target sensors. Control system actuators consist of $x-y$ emitter positioners (or hexapods), and phase controllers. An array with $n$ emitters has $4 \cdot n-6$ phase taps, $2 \cdot n-3$ IRFPA target sensors, and [6. $(n-2)+8 \cdot n-12]$ sixDOF relative position sensors. A preliminary assessment of controllability in progress, based on a baseline optical model of an array constructed using the principles described in preceding sections. The remainder of this paper is devoted to describing an optical model suitable for incorporating phase perturbations that are expected to exist across such an array. $^{32}$

\subsection{Time averaged intensity of Gaussian beams}

The resulting wave fronts from the fiber amplifiers can be modeled as a Gaussian beam. At any point in space, the time averaged intensity is given in terms of the symbols listed in Table 1 by:

$$
I=c \epsilon_{0} \int\left[E_{0}^{2}\left(\frac{w_{0}}{w(z)}\right)^{2} e^{-k r^{2} \operatorname{Im}\left[\frac{1}{q(z)}\right]} \cos ^{2}\left(k \frac{r^{2}}{2} R e\left[\frac{1}{q(z)}\right]\right) \cos ^{2}\left(\tan ^{-1}\left[\frac{z}{z_{0}}\right]\right) \cos ^{2}(k z+\omega t)\right]
$$

Where $1 / q(z)$ is given by:

$$
\frac{1}{q(z)}=\frac{1}{z\left(1+\frac{z^{2}}{z_{0}^{2}}\right)}+\frac{i \lambda}{\pi w_{0}^{2}\left(1+\frac{z^{2}}{z_{0}^{2}}\right)}
$$

Table 1. Terms used in Eq. (8) and throughout this paper.

\begin{tabular}{|c|c|c|}
\hline Symbol & Interpretation & Units \\
\hline$I$ & Time averaged intensity of beam & $\mathrm{W} / \mathrm{m}$ \\
\hline$c$ & Speed of light in a vacuum & $\mathrm{m} / \mathrm{s}$ \\
\hline$\varepsilon_{0}$ & Permittivity of free space & $\mathrm{F} / \mathrm{m}$ \\
\hline$E_{0}$ & Initial beam amplitude & $\mathrm{N} / \mathrm{C}$ \\
\hline$w_{0}$ & Beam waist & $\mathrm{m}$ \\
\hline$z$ & Forward propagation distance & $\mathrm{m}$ \\
\hline$r$ & Radial propagation distance & $\mathrm{m}$ \\
\hline$w(z)$ & Spot size & $\mathrm{m}$ \\
\hline$k$ & Wave number & $\mathrm{m}$ \\
\hline$q(z)$ & Complex radius of curvature & $\mathrm{m}$ \\
\hline$R(z)$ & Radius of curvature & $\mathrm{m}$ \\
\hline$\omega$ & Radial frequency & $\mathrm{Hz}$ \\
\hline$t$ & Time & $\mathrm{s}$ \\
\hline
\end{tabular}


Multiple beams can be simulated and their interference analyzed by summing their individual amplitudes under the square. The beams can then be propagated through an arbitrary optical system to focus at infinity. This is modeled using the $\mathrm{ABCD}$ law for Gaussian beams and is given by:

$$
q_{2}(z)=\frac{A q_{1}(z)+B}{C q_{1}(z)+D}
$$

Where $A, B, C$, and $D$ are the elements of the system matrix for the optical system. This creates an entirely new beam with different characteristics. As shown in Fig. 8, the incoming beam strikes the thin lens at $z=0.01$ and comes to a focus at $z=0.03$

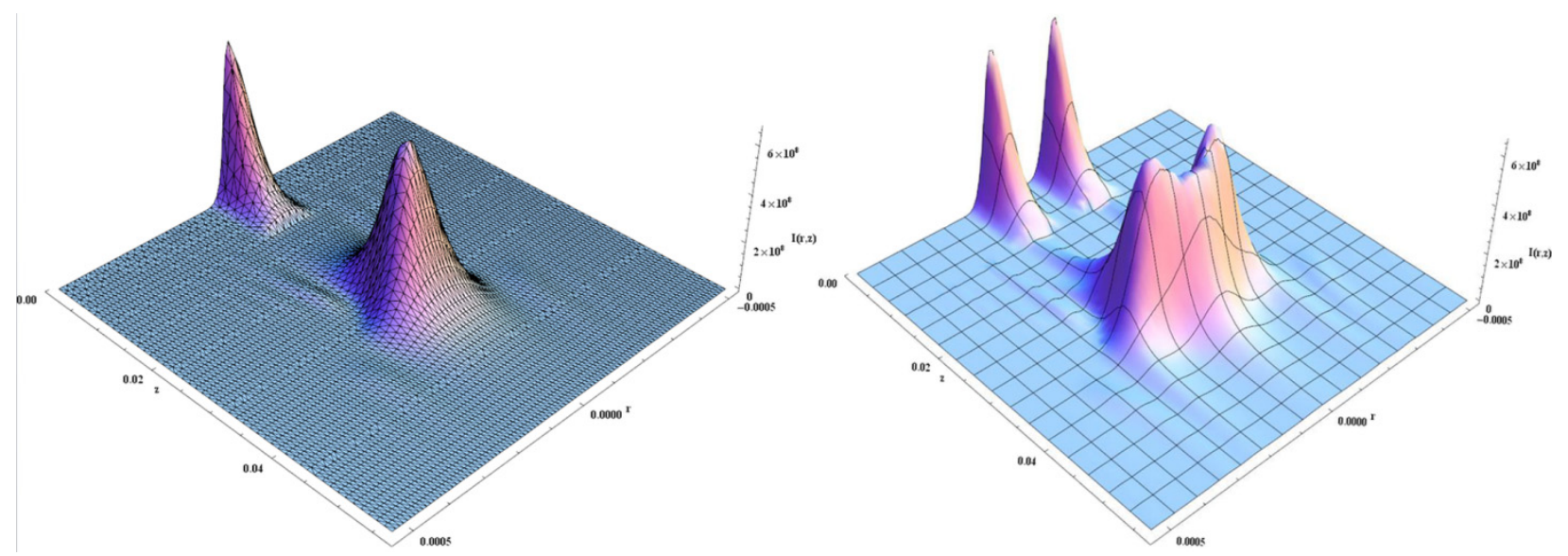

Figure 8. Left: High-resolution intensity pattern of a single Gaussian beam incident on a thin lens, based on the optical model of Eq. (8). Right: Lower-resolution model results of two Gaussian beams incident on a thin lens, showing initial combination of power in the focused signal.

The optical system used for the final control scheme simulations was a Fresnel lens. This is optically identical in the far field to a plano-convex lens. Thus, the transfer matrix for a plano-convex lens was used in the calculation to determine the system matrix for the modeled optical system.

\subsection{Inclusion of Phase Perturbations in the Optical Model}

To model possible non-mechanical phase drift, a time-varying phase term is added to the Gaussian wave front. To simulate a measurement from the phase tap, the calculated phase in the nominal location of the phase tap is determined for the true phase, and Gaussian noise is added to the calculated value. A linear Kalman filter is an optimal filter to smooth the measurements and calculate the proper phase correction by the electronic phase control which is modeled by yet another phase term added to the wavefront.

For mechanical phase perturbations, an oscillatory directional term is added to the Gaussian wave front. The amplitudes and frequencies of these terms come from finite element analysis (FEA) simulations performed in Abaqus on the design. To simulate a measurement from the capacitive proximity sensors, the true angular deflection of each cell is sampled and Gaussian noise is added. A linear Kalman filter is not able to resolve the oscillations of the structure and as such, an unscented Kalman filter will replace it. One method of correction is electronic phase control. This method, however, is only applicable to small amplitude oscillations. It is appropriate for higher order modes.

First and perhaps even second order modes may be too large in amplitude to be corrected by electronic phase control. The trade-off is, of course, lower frequency oscillations. This allows for mechanical corrective techniques. Using micropositioners in the two beam-orthogonal directions, the beam can be "steered" mechanically back to the target. The micropositioners are modeled as ideal directional terms taking input from the unscented Kalman filter above. Consideration of mechanical limitations must be taken into account if a physical model is to be built.

\subsection{Generation of Phase Perturbation Terms}

In the simulation, several terms are added to the Gaussian wavefront equation to introduce various sources of phase perturbation. These additions are listed and described in Table 2. 
Table 2. Phase Perturbation Terms

\begin{tabular}{|l|l|l|}
\hline Type of Perturbation & Simulation Method & Real-World Analog \\
\hline Optical Axis Deflection & $\begin{array}{l}\text { A rotational matrix transformation is } \\
\text { applied. }\end{array}$ & $\begin{array}{l}\text { First and second mode vibrations } \\
\text { of the structure. }\end{array}$ \\
\hline $\begin{array}{l}\text { Optical Axis Correction (large } \\
\text { amplitude) }\end{array}$ & $\begin{array}{l}\text { A rotational matrix is applied opposite } \\
\text { to that above and lagging behind to } \\
\text { simulate imperfect corrections. }\end{array}$ & $\begin{array}{l}\text { Micropositioners inside the cell } \\
\text { cavity. }\end{array}$ \\
\hline $\begin{array}{l}\text { Non-mechnical phase drift (i.e. } \\
\text { thermal, etc. })\end{array}$ & $\begin{array}{l}\text { A complex exponential with small } \\
\text { amplitude and slow phase is } \\
\text { multiplied by the wavefront. }\end{array}$ & $\begin{array}{l}\text { Thermal fluctuations and other } \\
\text { random phase disturbances. }\end{array}$ \\
\hline $\begin{array}{l}\text { Phase Drift and Small Amplitude } \\
\text { Optical Axis Correction }\end{array}$ & $\begin{array}{l}\text { A complex exponential with small } \\
\text { amplitude fast phase is multiplied by } \\
\text { the wavefront }\end{array}$ & $\begin{array}{l}\text { Electronic phase control in fiber } \\
\text { amplifiers. }\end{array}$ \\
\hline
\end{tabular}

\section{CONCLUSIONS}

Current laser phased array designs rely on an array-wide beamsplitter for phase feedback and phase control. In a scenario where an emitter cell is to be added to an existing emitter array, the beamsplitter would need to be replaced, and an array-wide control system recalibration would be required. As such, existing laser phased array designs are not so easily extensible. A conceptual design and phase control scheme is offered that is modular and extensible without requiring beamsplitter replacement or full-array re-calibrations. The technology would be useful if an array were to be constructed in stages, such as in low-Earth orbit. The basic concept for phase control is to align phases of all emitters in a reference plane that is normal to the target vector. A resultant target vector is determined from the combined information of all individual target sensors in the array, and within a common global coordinate system. The spatial relationship between adjacent target sensors is determined by 6-DOF position sensors, and the relative positioning is used to establish the global coordinate system. The position of target sensors and phase tap structures within the global coordinate system is determinable in control-time. Phase feedback is determined by the distance of each phase tap from the reference plane. Controllability of an array is being investigated, beginning with optical models that incorporate realistic sources of phase perturbations for individual emitters.

\section{ACKNOWLEDGEMENTS}

We gratefully acknowledge funding from the NASA California Space Grant NASA NNX10AT93H in support of this research. We also gratefully acknowledge funding from the NASA Innovative Advanced Concepts grant NNH15ZOA001N in support of this research.

\section{REFERENCES}

\section{DE-STAR and DE-STARLITE}

[1] Lubin, P., Hughes, G.B., Bible, J., Bublitz, J., Arriola, J., Motta, C., Suen, J., Johansson, I., Riley, J., Sarvian, N., Clayton-Warwick, D., Wu, J., Milich, A., Oleson, M., Pryor, M., Krogen, P., Kangas, M., and O’Neill, H. “Toward Directed Energy Planetary Defense," Optical Engineering, Vol. 53, No. 2, pp 025103-1 to 025103-18 (Feb, 2014), doi: 10.1117/1.OE.53.2.025103.

[2] Hughes, G.B., Lubin, P., Bible, J., Bublitz, J., Arriola, J., Motta, C., Suen, J., Johansson, I.E., Riley, J., Sarvian, N., Wu, J., Milich, A., Oleson, M., and Pryor, M. "DE-STAR: phased-array laser technology for planetary 
defense and other scientific purposes," Nanophotonics and Macrophotonics for Space Environments VII, edited by Edward W. Taylor, David A. Cardimona, Proc. of SPIE Vol. 8876, 88760J (Aug, 2013).

[3] Kosmo, K., Pryor, M., Lubin, P., Hughes, G.B., O’Neill, H., Meinhold, P., Suen, J., C., Riley, J., Griswold, J., Cook, B.V., Johansson, I.E., Zhang, Q., Walsh, K., Melis, C., Kangas, M., Bible, J., Motta, Brashears, T., Mathew, S. and Bollag, J. "DE-STARLITE - a practical planetary defense mission," Nanophotonics and Macrophotonics for Space Environments VIII, edited by Edward W. Taylor, David A. Cardimona, Proc. of SPIE Vol. 9226 (Aug, 2014).

[4] Kosmo, K., Lubin, P., Hughes, G.B., Griswold, J., Zhang, Q. and Brashears, T. "Directed Energy Planetary Defense," Aerospace Conference 2015 IEEE Proceedings, 7-14 March 2015, ISBN: 978-1-4799-5379-0 (Mar, 2015).

[5] Bible, J., Bublitz, J., Johansson, I.E., Hughes, G.B., and Lubin, P. "Relativistic Propulsion Using Directed Energy," Nanophotonics and Macrophotonics for Space Environments VII, edited by Edward W. Taylor, David A. Cardimona, Proc. of SPIE Vol. 8876, 887605 (Aug, 2013).

[6] Riley, J., Lubin, P., Hughes, G.B., O’Neill, H., Meinhold, P., Suen, J., Bible, J., Johansson, I.E., Griswold, J. and Cook, B. "Directed energy active illumination for near-Earth object detection," Nanophotonics and Macrophotonics for Space Environments VIII, edited by Edward W. Taylor, David A. Cardimona, Proc. of SPIE Vol. 9226 (Aug, 2014).

[7] Hughes, G.B., Lubin, P., Meinhold, P., O’Neill, Brashears, T., Zhang, Q., Griswold, J., Riley, J., and Motta, C. "Stand-off molecular composition analysis," Nanophotonics and Macrophotonics for Space Environments IX, edited by Edward W. Taylor, David A. Cardimona, Proc. of SPIE Vol. 9616 (Aug, 2015).

Fiber Laser Amplifiers and Laser Phased Arrays

[8] Zervas, M.N. and Codemard, C.A. "High power fiber lasers: a review." IEEE Journal of Selected Topics in Quantum Electronics, vol. 20, no. 5, pp. 219-241 (2014).

[9] Wagner, T.J. "Fiber laser beam combining and power scaling progress: Air Force Research Laboratory Laser Division." SPIE LASE, pp. 823718-823718. Proc. of SPIE vol. 8237 (Feb, 2012).

[10] Vorontsov, M.A., Weyrauch, T., Beresnev, L.A., Carhart, G.W., Liu, L. and Aschenback, K. “Adaptive Array of Phase-Locked Fiber Collimators: Analysis and Experimental Demonstration," IEEE Journal of Selected Topics in Quantum Electronics, vol. 15, 269 (2009).

[11] Liu, H., He, B., Zhou, J., Dong, J., Wei, Y., \& Lou, Q. "Coherent beam combination of two nanosecond fiber amplifiers by an all-optical feedback loop.” Optics Letters, 37(18), 3885-3887 (2012).

[12] Su, R., Zhou, P., Wang, X., Zhang, H., \& Xu, X. "Active coherent beam combining of a five-element, $800 \mathrm{~W}$ nanosecond fiber amplifier array.” Optics Letters, 37(19), 3978-3980 (2012)

[13] Yu, C.X., Augst, S.J., Redmond, S.M., Goldizen, K.C., Murphy, D.V., Sanchez, A., and Fan, T.Y. "Coherent combining of a 4 kW, eight-element fiber amplifier array." Optics Letters, 36(14), 2686-2688 (2011).

\section{Heterodyne Control of Laser Phased Arrays}

[14] Anderegg, J., Brosnan, S., Cheung, E., Epp, P., Hammons, D., Komine, H., Weber, M. and Wickham, M. "Coherently coupled high-power fiber arrays," in Lasers and Applications in Science and Engineering, edited by Andrew J. W. Brown; Johan Nilsson; Donald J. Harter; Andreas Tünnermann, Proc. of SPIE, vol. 6102, pp. 61020U-61020U (Feb, 2006).

[15] Cheung, E.C., Ho, J.G., Goodno, G.D., Rice, R.R., Rothenberg, J., Thielen, P., Weber, M. and Wickham, M. "Diffractive-optics-based beam combination of a phase-locked fiber laser array." Optics letters, 33(4), 354-356 (2008).

[16] Goodno, G. D., McNaught, S. J., Rothenberg, J. E., McComb, T. S., Thielen, P. A., Wickham, M. G. and Weber, M. E. Active phase and polarization locking of a $1.4 \mathrm{~kW}$ fiber amplifier. Optics letters, 35(10), 1542-1544 (2010).

\section{SPGD Control of Laser Phased Arrays}

[17] Vorontsov, M., Carhart, G.W. and Ricklin, J.C. "Adaptive Phase Distortion Correction Based On Parallel Gradient Descent Optimization," Optics Letters, 22(12), pp. 907-909 (1997).

[18] Liu, L., Vorontsov, M.A., Polnau, E., Weyrauch, T. and Beresnev, L.A. "Adaptive Phase-Locked Fiber Array with Wavefront Phase Tip-Tilt Compensation using Piezoelectric Fiber Positioners," in: Atmospheric Optics: Models, 
Measurements, and Target-in-the-Loop Propagation, edited by Stephen M. Hammel, Alexander M. J. van Eijk, Michael T. Valley, Mikhail A. Vorontsov, Proc. of SPIE, Vol. 6708, pgs. 67080K-1 - 67080K-12 (2007).

[19] Zhou, P., Liu, Z., Wang, X., Ma, Y., Ma, H. and Xu, X. "Coherent Beam Combination of Two Dimensional High Power Fiber Amplifier Array Using Stochastic Parallel Gradient Descent Algorithm." Applied Physics Letters, Vol. 94, No. 231106, pgs. 231106-1 - 231106-3 (2009).

[20] Zhou, P., Liu, Z., Wang, X., Ma, Y., Ma, H., and Xu, X. "Coherent Beam Combining of Two Fiber Amplifiers Using Stochastic Parallel Gradient Descent Algorithm." Optics \& Laser Technology, Vol. 41, pp. 853 - 856 (2009).

Target-In-The-Loop Control of Laser Phased Arrays

[21] Liu, L. and Vorontsov, M. A. "Phase-locking of tiled fiber array using SPGD feedback controller," In Target-inthe-Loop: Atmospheric Tracking, Imaging, and Compensation II, edited by Michael T. Valley, Mikhail A. Vorontsov, Proc. of SPIE, vol. 5895, pp. 58950P-58950P (Aug, 2005).

[22] Lachinova, S. L., and Vorontsov, M. A. "Laser beam projection with adaptive array of fiber collimators. II. Analysis of atmospheric compensation efficiency," Journal of the Optical Society of America A, 25(8), 1960-1973 (2008).

\section{Optical Alignment}

[23] Yu, N., Genevet, P., Aieta, F., Kats, M.A., Blanchard, R., Aoust, G., Tetienne, J.Pl, Gaburro, Z. and Capasso, F. "Flat Optics: Controlling Wavefronts with Optical Antenna Metasurfaces." IEEE Journal of Selected Topics in Quantum Electronics, vol. 19, no. 3 (May/June 2013).

[24] Hughes, G.B., Lubin, P., Griswold, J., Bozinni, D., O’Neill, H., Meinhold, P., Suen, J., Bible, J., Riley, J., Johansson, I., Pryor, M. and Kangas, M. "Optical modeling for a laser phased-array directed energy system," Nanophotonics and Macrophotonics for Space Environments VIII, edited by Edward W. Taylor, David A. Cardimona, Proc. of SPIE Vol. 9226 (Aug, 2014).

[25] Hadjiyska, E., Hughes, G.B., Lubin, P., Taylor, S., Hartong-Redden, R. and Zierten, J. "The Transient Optical Sky Survey data pipeline," New Astronomy, vol. 19, pp. 99-108 (2013).

[26] R. Y. Tsai. “A Versatile Camera Calibration Technique for 3D Machine Vision”, IEEE Journal of Robotics \& Automation, vol. RA-3, no. 4, pp. 323-344 (Aug 1987).

[27] Zhang, Z. "A flexible new technique for camera calibration." IEEE Transactions on Pattern Analysis and Machine Intelligence, 22(11), pp. 1330-1334 (2000).

[28] Sun, Q., Hou, Y., Tan, Q. and Li, G. "A Flexible Calibration Method Using the Planar Target with a Square Pattern for Line Structured Light Vision System,” PLOS One, vol. 9, no. 9, pp. e106911 (Sep 2014).

[29] Kannala, J. and Brandt, S.S. "A generic camera model and calibration method for conventional, wide-angle, and fish-eye lenses," IEEE Transactions on Pattern Analysis and Machine Intelligence, 28(8), pp. 1335-1340 (2006).

[30] Hughes, G.B. "Algorithms for Sensor Chip Alignment to Blind Datums," Journal of Electronic Imaging, vol. 15, no. 3, pp. 033003-1 to 033003-13 (Jul-Sep 2006).

[31] Sison, C.A., Hughes, G.B., Lubin, P., Meinhold, P. and Suen, J. “A high-precision six degree-of-freedom relative position sensor" (in preparation, Jun 2015).

[32] Hughes, G.B., Lubin, P., Griswold, J., Bozinni, D., O’Neill, H., Meinhold, P., Suen, J., Bible, J., Riley, J., Johansson, I., Pryor, M. and Kangas, M. "Optical modeling for a laser phased-array directed energy system (Invited Paper)," Nanophotonics and Macrophotonics for Space Environments VIII, edited by Edward W. Taylor, David A. Cardimona, Proc. of SPIE Vol. 9226 (Aug, 2014). 